\title{
A qualidade do pré-natal no Brasil
}

\author{
Quality of the antenatal care in Brasil
}

João Luiz Pinto e Silva ${ }^{1}$, José Guilherme Cecatti ${ }^{1}$, Suzanne Jacob Serruya²

\section{0 pré-natal atual}

A maioria dos programas atuais de atenção pré-natal é originada de modelos desenvolvidos em países ocidentais, nas primeiras décadas do século passado.Embora parcela significativa tenha calendário e conteúdo similares, diferem quanto ao tipo de profissionais envolvidos, às práticas recomendadas e realizadas, além de diferentes taxas de adesão de mulheres ${ }^{1,2}$

Vários autores têm realizado proficuo debate sobre os modelos de pré-natal, discutindo principalmente as bases científicas sobre as quais se construíram e os resultados sobre a saúde materna e perinatal $1^{3-5}$. Com intenção de conduzir essa discussão, estudo sistemático avaliou diferentes modelos e suas repercussões, analisando um conjunto de variáveis, baseado em intervenções com evidências, e que constituam um modelo, que otimize recursos e não acrescente riscos às gestantes que não apresentem complicações ${ }^{6}$.

No Brasil, a assistência pré-natal tem apresentado importantes diferenciais em numerosos aspectos, que vão do acesso, ao número de consultas, passando por seus conteúdos, periodicidade e profissionais de saúde envolvidos em sua realização ${ }^{7}$. A realidade do panorama obstétrico, confrontada com as taxas altas de morbimortalidade materna e perinatal, induziram o Ministério da Saúde em $2000^{8}$, a lançar uma estratégia de ação, com o objetivo de definir um modelo nacional que normatizasse as ações assistenciais relacionadas, conjugando esforços para melhorar os resultados observados. O programa proposto, denominado Programa de Humanização do Pré Natal ( PHPN) ${ }^{8}$, trouxe em seu bojo a discussão sobre as práticas pré-natais e suas bases conceituais, em consonância com os modelos utilizados em todo o mundo.

\section{Programa de Humanização do Pré-natal (PHPN)}

O PHPN em linhas gerais recomendou primeira consulta até o $4^{\circ}$ mês de gravidez e a adoção dos seguintes procedimentos: seis consultas, no mínimo, para cada mulher: preferencialmente uma no primeiro trimestre, duas no segundo e três no terceiro; uma consulta no puerpério, até quarenta dias após o parto; exames laboratoriais: tipagem de sangue, hemoglobina/hematócrito, VDRL, urina de rotina, glicemia de jejum, todos realizados na primeira consulta e os quatro últimos, repetidos próximo a trigésima semana da gestação; teste de HIV oferecido na primeira consulta, em municípios de população acima de 50.000; aplicação de vacina antitetânica, até a dose imunizante (segunda), ou reforço para as já imunizadas; classificação continuada de risco gestacional e atividades educativas.

Até então, não haviam estudos conclusivos sobre o número mínimo ou ideal para se lograr os resultados esperados ${ }^{6,9}$. A maioria recomendava uma consulta no primeiro trimestre, considerada precoce, e de impacto, variando a partir daí, sobre o número ideal e sua periodicidade.Como o país apresentava em média quatro consultas por mulher que tinha seu parto na rede do SUS, supôs-se que um incremento de $50 \%$, ou seja mais duas consultas, seria uma proposta viável, sem perder a perspectiva de diferenças e dificuldades regionais. 
Na pesquisa da OMS de $2002^{10,11}$, recomendou-se o total de quatro consultas para cada mulher, todas durante a gravidez, que foram consideradas satisfatórias pelas mulheres entrevistadas por métodos quantitativos e qualitativos, com grupos focais e em profundidade ${ }^{12}$. Ressalte-se que uma preocupação destacada foi pelo número e espaçamento das consultas, com o relacionamento com os profissionais, e a com a necessidade de se obter mais informações sobre vários temas durante a gravide $z^{9}$. A discussão sobre o modelo pontuou questões sobre o formato geral, principalmente sobe os critérios que envolviam o número de consultas, sua oportunidade, e a escolha de recursos de apoio diagnóstico, principalmente a ultrassonografia. De maneira geral, considerou-se o formato razoável, considerando -se as diferenças regionais, admitindo-se que as exigências básicas eram adequadas para a maioria dos municipios, e que os acréscimos possiveis, dependeriam de recursos e desempenho de cada um deles.

O modelo brasileiro comparado com o proposto pela OMS, formatado para um estudo aleatorizado, e que considerou seleção mais restrita de exames e de consultas, é ligeiramente mais abrangente.No formato do PHPN por exemplo, propõe-se a detecção do anti-HIV e o rastreamento do diabetes, diferença que poderia estar apoiada em decisões operacionais em termos de custo e capacidade instalada, e não epidemiológicas, e no fato de que o estudo internacional restringe a execução de testes àqueles com evidências científicas. Outras diferenças aparecem no método aplicado para a realização dos testes comuns aos dois modelos: ABO-Rh, Hb, VDRL e análise de urina.No PHPN se recomenda a realização de todos os exames à primeira consulta, e próximo da $30^{a}$ semana a repetição de alguns.

No modelo da OMS, há diferentes abordagens, ao longo das quatro consultas, a depender de sinais e sintomas da presença de complicações gestacionais, ou da suspeita delas. Para a realização do VDRL, a OMS recomenda o teste rápido e o tratamento imediato no momento da consulta.A estratégia procura facilitar acesso para todas as mulheres, liga o exame ao resultado imediato e ao tratamento, e propicia aos profissionais melhor organização e internalização das intervenções.A alternativa proporciona ainda facilidades à paciente, no sentido de conseguir a "solicitação" do exame, sua realização sem necessidade de novo deslocamento, a apresentação do resultado, e início imediato de eventual tratamento, vantagens acumuladas na mesma consulta.

A razão para esta análise comparativa é que o estudo conduzido pela OMS,fornece importantes elementos para a compreensão da questão da assistência pré-natal e para a discussão das estratégias que garantam maior efetividade.Sua proposta de conjugação de diagnóstico e tratamento, além de garantir resultados, permite melhor gestão da assistência e satisfação por parte das clientes atendidas. Sua sistematização para um país como o nosso, com grandes desigualdades regionais, distribuídas por mais de cinco mil municípios, igualmente como se faz para o PHPN, recomenda uma ampla discussão que considere aspectos ligados à operacionalização, custo beneficio e principalmente, os resultados maternos e perinatais.

\section{Avaliações do programa brasileiro (PHPN)}

A avaliação preliminar da experiência de implantação do programa no Brasil, feita por estudo descritivo, populacional, com informações referentes aos anos de 2001 e 2002, mostrou $72 \%$ de adesão dos municípios com apresentação de produção, o que permitiu a formatação de banco de dados com 720.871 mulheres $^{7}$. Até então, sua avaliação havia sido feita por iniciativas isoladas de alguns municipios, como os trabalhos de Trevisan et $\mathrm{al}^{12}$, em 2002 em Caxias do Sul (RS) e de Coutinho et al. ${ }^{13}$ em 2003 em Juiz de Fora (MG).

Destacaram estes autores, que a assistência ao pré-natal de baixo risco nestes municípios, apesar da boa cobertura, deveria ser revista, considerando o baixo cumprimento das normas do programa oficial, principalmente no que se relacionava à captação tardia para a primeira consulta e o não cumprimento do número estipulado de consultas e dos exames complementares recomendados.Ambos enfatizaram, ademais, a necessidade de rever qualitativamente a assistência oferecida, e promover avaliações periódicas, como instrumentos imprescindiveis para seu aperfeiçoamento.

A avaliação preliminar dos dados gerados nestes dois anos de implantação, através do Programa SISPRENATAL, mostrou que apenas uma pequena parcela das gestantes cadastradas cumpriu 
integralmente os critérios estabelecidos em seus objetivos, sendo que a maioria apresentou assistência desarticulada e parcial.Apenas $25 \%$ conseguiram o número de seis consultas ou mais, chamando atenção para as dificuldades em realizar os exames laboratoriais e a consulta puerperal, dificuldades que sinalizam para a necessidade de rever sua dinâmica de realização ${ }^{7}$.

Agrega esta avaliação, a conclusão de que, apesar dos indicadores de qualidade mostrarem melhora de uma ano para outro, permaneceram baixos os percentuais registrados, ratificando a necessidade de permanentes ajustes, avaliações e novas intervenções, particularmente nas regiões mais carentes como Norte e Nordeste. Registra-se desta maneira, que a inadequação da utilização dos cuidados pré-natais, está associada com fatores de natureza social, agravada por desigualdades sócio-econômicas, o que complica mais as soluções para a construção de um modelo apropriado e de melhores resultados.

As estatísticas permanentemente preocupantes, de morbimortalidade materna e perinatal nos países em desenvolvimento como o Brasil, estão fortemente entrelaçadas com a não cumprimento de cuidados maternos e perinatais de boa qualidade.A tradição de se adotar, sem uma crítica pertinente, programas recomendados em países desenvolvidos, têm demonstrado que não resultou em beneficios para nossas populações.Apesar de se buscar melhorar os cuidados ante-natais, com a incorporação sistemática de novas técnicas e procedimentos, a não identificação correta das intervenções efetivas, cientificamente comprovadas, tem contribuído para dispersar os parcos recursos disponiveis, que historicamente contemplam os cuidados da nossas gestantes.

O PHPN ainda necessita avançar mais em sua execução, para melhor se conhecer o seu verdadeiro impacto na saúde das mulheres. Seu confronto com outros modelos, particularmente com o da OMS, em muitos aspectos, com características muito semelhantes, mostra que sua implementação e permanente revisão podem proporcionar resultados superiores ao padrão atual, permitindo avanços substanciais nos resultados maternos e perinatais.

\section{Referências}

1. Bergsjo P, Villar J. Scientific basis for the content of routine antenatal care. II. Power to eliminate or allevite adverse newborn outcomes: come especial conditions and examinations. Acta Obstet Gynecol Scand. 1997;76(1):15-25.

2. Lumbiganon P. Appropriate technology: antenatal care. Int J Gynaecol Obstet. 1998;63 Suppl 1:S91-5.

3. Faúndes A, Pinotti JA, Cecatti JG. Atendimento pré-natal: assistência obstétrica primária; quais as necessidades no Brasil? J Bras Med. 1987;52(3):38-54.

4. Villar J, Garcia P, Walker G. Routine antenatal care. Curr Opin Obstet Gynecol. 1993;5(5):688-93.

5. Villar J, Carroli G, Khan-Neelofur D, Piaggio G, Gülmezoglu M. Patterns of routine antenatal care for lowrisk pregnancy. Cochrane Database Syst Rev. 2001;(4):CD000934.

6. Carroli G, Rooney C, Villar J. How effective is antenatal care in preventing maternal mortality and serious morbidity? An overview of the evidence. Pediatr Perinat Epidemiol. 2001;15 Suppl 1:1-42.

7. Serruya SJ, Lago TG, Cecatti JG. Avaliação preliminar do programa de humanização no pré-natal e nascimento no Brasil. Rev Bras Ginecol Obstet. 2004;26(7):517-24.

8. Ministério da Saúde. Secretaria de Políticas de Saúde. Programa de humanização no pré-natal e nascimento. Brasília; 2000.

9. Villar J, Ba'aqeel H, Piaggio G, Lumbiganon P, Miguel Belizán J, Farnot U, et al. WHO antenatal care randomised trial for the evaluation of a new model of routine antenatal care. Lancet. 2001;357(9268):1551-64.

10.World Health Organization. WHO Programme to map best reproductive health practice. WHO Antenatal Care Randomized Trial: manual for the implementation of the new model. Geneva: World Health Organization; 2002.

11.Nigenda G, Romero M, Langer A. Refexiones metodológicas en torno a un estudio cuali-cuantitativo sobre la percepción de la calidad de la atención prenatal en países en desarrollo. Experiencias inovadoras en salud reproductiva. La complementación de las ciencias médicas y sociales. Buenos Aires: CEDES; 2002. p. 21-44.

12.Trevisan MR, De Lorenzi DRS, Araujo NM, Ésber K. Perfil da assistência pré-natal entre usuárias do Sistema Único de Saúde em Caxias do Sul. Rev Bras Ginecol Obstet. 2002;24(5):239-9.

13.Coutinho T, Teixeira MTB, Dain S, Sayd JD, Coutinho LM. Adequação do processo de assistência pré-natal entre usuárias do Sistema Único de Saúde em Juiz de Fora, MG. Rev Bras Ginecol Obstet. 2003;25(10):717-24. 